\title{
Scattering of Monatomic Gas Molecules on Vertically Aligned Single-Walled Carbon Nanotubes
}

\author{
Ikuya Kinefuchi ${ }^{a}$, Keisuke Nakazono ${ }^{a}$, Yushi Harada ${ }^{a}$, Kei Ishikawa ${ }^{a}$, \\ Junichiro Shiomi ${ }^{\mathrm{a}}$, Shu Takagi ${ }^{\mathrm{b}, \mathrm{a}}$, Shigeo Maruyama ${ }^{\mathrm{a}}$, and Yoichiro Matsumoto ${ }^{\mathrm{a}}$ \\ ${ }^{a}$ Department of Mechanical Engineering, The University of Tokyo, 7-3-1 Hongo, Bunkyo-ku, Tokyo 113-8656, Japan \\ ${ }^{b}$ Computational Science Research Program, RIKEN, 2-1 Hirosawa, Wako, Saitama 351-0198, Japan
}

\begin{abstract}
The scattering process of He molecules on vertically aligned single-walled carbon nanotubes (VA-SWNTs) was investigated using the molecular beam technique. The accommodation coefficients for VA-SWNT films on quartz substrates at room temperature are remarkably high compared to those for bare surfaces, demonstrating the effectiveness of the surface modification technique with VA-SWNT films for enhancing the energy transfer between gas molecules and surfaces. The enhanced energy transfer suggests that gas molecules can easily penetrate deep into the films because of their high porosity and suffer multiple collisions with bundles of SWNTs. The less effective energy accommodation at elevated temperatures, however, implies that the average number of collisions which gas molecules undergo before leaving the films is not large enough to maintain the perfect accommodation even at high temperature.
\end{abstract}

Keywords: Gas-surface interaction, Molecular beam, Carbon nanotubes

PACS: 34.35.+a, 68.49.Df, 81.07.De

\section{INTRODUCTION}

Single-walled carbon nanotubes (SWNTs) [1] have recently drawn increasing attention from scientific and technological points of view owing to their outstanding physical properties [2] such as high thermal conductivity and optical anisotropy. The chemical vapor deposition (CVD) with adequate conditions yields the uniform films consisting of vertically aligned SWNTs (VA-SWNTs) over large areas of substrates [3]. Because of their unique morphology, the films of VA-SWNTs offer the useful platform to investigate the physical properties and the growth mechanism of SWNTs. Moreover, the films themselves are quite attractive materials for potential applications.

We focused on the potential application of VA-SWNT films for the surface modification to control gas-surface interactions in rarefied gas flows since their nanometer-sized fin-like structure would increase the number of collisions between gas molecules and surfaces. A number of researchers have reported on the ad/desorption kinetics and energetics of gas molecules on SWNTs [4] since the development of the potential SWNT-based applications such as gas storage devices and nanosensors requires information on the characteristics of gas-SWNT interactions that these devices rely on. There are few studies, however, on the dynamic process of these interactions (i.e. the scattering process) [5], which determines the performance of the nanometer-sized fins made of VA-SWNTs. The detailed understanding of the scattering process is also in great demand to predict the growth kinetics of VA-SWNT films by CVD methods, where feedstock gas molecules have to diffuse through the growing films to reach the catalyst nanoparticles on the substrates [6].

We therefore have been conducting the scattering experiments of gas molecules on VA-SWNT films using the molecular beam technique [7]. The angular distributions and the time-of-flight distributions of scattered molecules were measured to analyze the scattering mechanism, especially the energy accommodation process. At present, we limit ourselves to the measurement with helium molecular beam because of the simplicity of the analysis. The results obtained from our experiment, however still give us the insight into the distinctive scattering process caused by the unique structure. We found that the surface modification with VA-SWNTs significantly enhances the energy transfer between gas molecules and surfaces and makes the energy accommodation coefficient of helium, which tends to be small even for contaminated surfaces [8-10], close to unity. 


\section{EXPERIMENT}

The samples used were the films of VA-SWNTs grown on quartz glass substrates by alcohol CVD method [3]. The transmission electron microscopy observation revealed that SWNTs in the films have an average diameter of about $2 \mathrm{~nm}$ and come together to form bundles, which typically contain less than ten SWNTs per bundle [11]. Figure 1 shows the secondary electron microscopy (SEM) images of the samples, showing that the thicknesses of them were approximately $0.1 \mu \mathrm{m}$ and $4 \mu \mathrm{m}$. The former (thinner) sample consists of SWNT bundles oriented randomly and does not have well-aligned structure. On the other hand, SWNT bundles align vertically in most part of the latter (thicker) sample except for the topmost layer, whose structure is similar to that of the thinner sample. These samples are estimated to have the porosity of $97 \%$ [6] although the detailed structure is still under debate.

We conducted experiments in an ultrahigh vacuum chamber equipped with a supersonic molecular beam source [12]. He molecular beam was formed by supersonic expansion from a $50 \mu \mathrm{m}$ diameter orifice at room temperature $(\sim 300 \mathrm{~K})$ and collimated through a three-stage differential pumping system so that the spot diameter at the sample surface became about $5 \mathrm{~mm}$. Before entering the main chamber, the beam was modulated by a two-slit chopper rotating at $100 \mathrm{~Hz}$ with $0.4 \%$ duty cycle. Figure 2 illustrates the configurations of the experiments. In most of the experiments, the as-grown samples (i.e. VA-SWNTs films on quartz substrates) heated at fixed temperatures between 300 and $800 \mathrm{~K}$ were exposed to the molecular beam. The scattered molecules were detected using a quadrupole mass spectrometer (QMS) mounted on a rotatable positioning assembly. In order to make it clear whether incident molecules penetrate deep into the film or are scattered back from its topmost layer, we also performed the experiments with the freestanding films, which were prepared by pealing off as-grown films from quartz substrates and transferring them onto the sample holders with a $4 \mathrm{~mm}$ diameter hole using the hot-waterassisted process [13]. The QMS, which was positioned on the back side of the sample, detected the transmitted molecules through the freestanding film. In this paper, we describe the experiments with as-grown films, while those with the freestanding films will be presented elsewhere.

The time-of-flight (TOF) distribution of the detected gas molecules can be described by the convolution integral

$$
S(t)=\int_{-\infty}^{\infty} R(t-\tau)\left[\gamma P_{\mathrm{dir}}(\tau)+(1-\gamma) P_{\text {ads }}(\tau)\right] d \tau,
$$

where $R(t)$ is the incident beam flux on the surface, and the terms inside the bracket represent the TOF distribution of the scattered molecules from the surface to the QMS. Note that $R(t)$ is a convolution of the chopper gate function and the TOF distribution of incident molecules and hence is a known distribution. We assumed that the TOF distribution of the scattered molecules follows a linear superposition of the direct inelastic scattering component $P_{\text {dir }}(t)$ and the trapping-desorption component $P_{\text {ads }}(t)$ with a weighting factor $\gamma(0 \leq \gamma \leq 1)$ [14]. These components

(a)
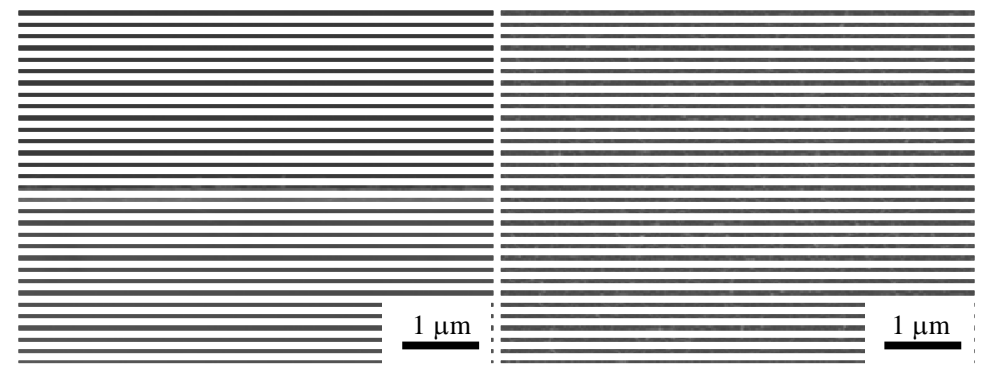

(b)

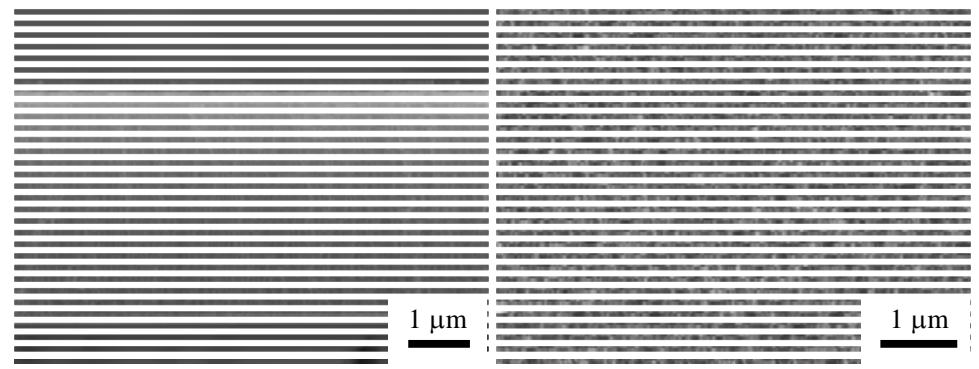

FIGURE 1. The SEM images of VA-SWNT films grown on quartz substrates: the side view (left) and the top view (right) of the films with thicknesses of $0.1 \mu \mathrm{m}$ (a) and $4 \mu \mathrm{m}$ (b). 
(a)

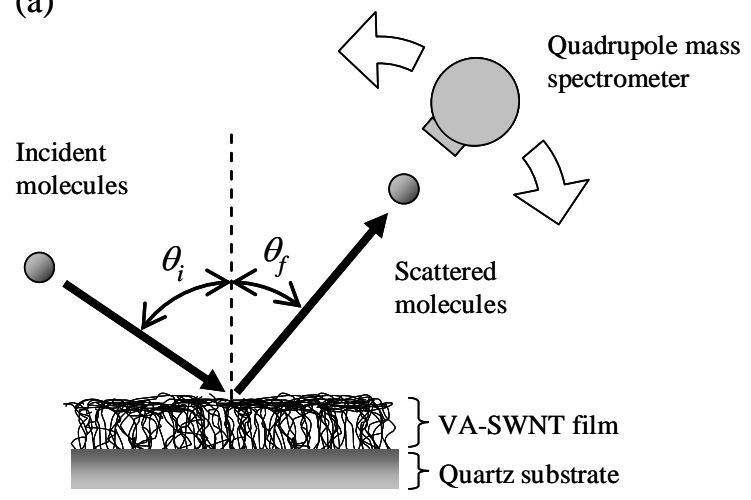

(b)

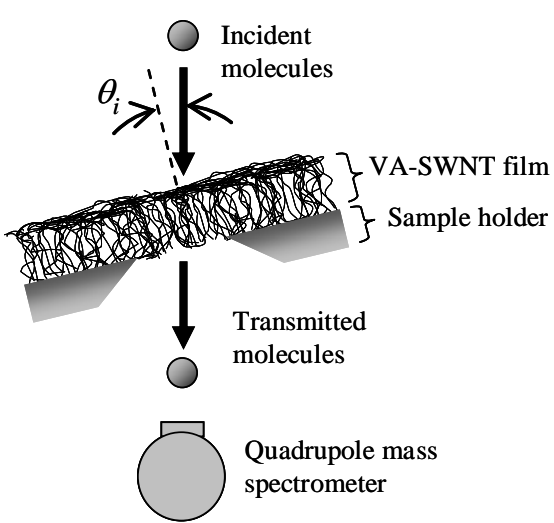

FIGURE 2. The schematic illustration of the experiments with the as-grown film on the quartz substrate (a) and the freestanding film transferred onto the sample holder (b).

are expressed as

$$
\begin{gathered}
P_{\text {dir }}(t)=\frac{c_{1}}{t^{4}} \exp \left[-\frac{1}{\alpha_{1}^{2}}\left(\frac{L}{t}-U\right)^{2}\right], \\
P_{\text {dir }}(t)=\frac{c_{2}}{t^{4}} \exp \left[-\frac{1}{\alpha_{2}^{2}}\left(\frac{L}{t}\right)^{2}\right],
\end{gathered}
$$

where $L$ is the distance between the surface and the QMS, $U$ the drift velocity, $\alpha_{1}$ and $\alpha_{2}$ the most probable speeds, and $c_{1}$ and $c_{2}$ the normalization constants. The mean translational energies for each component are given by

$$
\begin{gathered}
E_{1}=\frac{1}{2} m \alpha_{1}^{2} \cdot \frac{\sqrt{\pi} S\left(15 / 4+5 S^{2}+S^{4}\right)[1+\operatorname{erf}(S)]+\left(1 / 2+S^{2}\right)\left(4+S^{2}\right) \exp \left(-S^{2}\right)}{\sqrt{\pi} S\left(3 / 2+S^{2}\right)[1+\operatorname{erf}(S)]+\left(1+S^{2}\right) \exp \left(-S^{2}\right)}, \\
E_{2}=m \alpha_{2}^{2},
\end{gathered}
$$

where $m$ is the mass of a gas molecule, and $S$ the speed ratio defined by $U / \alpha_{1}$. We can thus evaluate the mean translational energy of scattered molecules with

$$
E_{f}=\gamma E_{1}+(1-\gamma) E_{2} .
$$

We estimated the energy accommodation coefficients $\alpha$ from the scattering experiments although our measurements were limited to the in-plane scattering, where the scattering directions of molecules are on the plane spanned by the normal vector of the surface and the velocity vector of the incident molecule. As described blow, the angular dependence of in-plane scattering intensities in most cases follows the cosine distribution. Hence, we estimated the mean translational energy of molecules scattered over all directions $\bar{E}_{f}$ by assuming that the scattering intensity follows the cosine distribution even in the out-of-plane and that the mean translational energy of scattered molecules depends only on the angle between the scattering direction and the surface normal. The energy accommodation coefficient is defined by

$$
\alpha=\left(\bar{E}_{f}-\bar{E}_{i}\right) /\left(2 k_{B} T_{s}-\bar{E}_{i}\right),
$$

where $\bar{E}_{i}$ is the mean translational energy of the incident beam, $T_{s}$ the surface temperature. Note that $2 k_{B} T_{s}$ is the mean translational energy of desorbing gas molecules from the equilibrium state on the surface.

\section{RESULTS AND DISCUSSION}

Figure 3 summarizes the results of the scattering experiments for the pre-growth quartz substrate, on which CoMo catalyst particles with diameters of $1-2 \mathrm{~nm}$ were well dispersed without agglomeration [15]. The sample was at room temperature during the experiment. The scattering distribution for the smaller incident angle $\theta_{i}$, which is measured from the surface normal, exhibits the diffusive (cosine) distribution. The translational energies of the 
scattered molecules, however, are far from that corresponding to the perfect accommodation and rather close to the incident beam energy in spite of the diffusive scattering distribution. These results suggest that the atomically rough surface makes the scattering direction completely random and thus independent of the incident angle even though gas molecules interact with the surface for insufficient time to reach the equilibrium state. The scattering distribution for the larger incident angle $\theta_{i}$ deviates from the cosine distribution, indicating the incomplete accommodation.

Figure 4 shows the results of the scattering experiments for the VA-SWNT film with a thickness of $4 \mu \mathrm{m}$ at room temperature. The intensity of scattered molecules from the VA-SWNT film exhibits the cosine distribution even for the large incident angles in contrast to that from the quartz substrate. Moreover, the mean translational energies of scattered molecules are considerably close to the value corresponding to the perfect accommodation $2 k_{B} T_{s}$, demonstrating the effectiveness of the surface modification technique with VA-SWNT films for the enhancement of the energy transfer between gas molecules and surfaces. For large incident angles $\theta_{i}$ (i.e. small grazing angles of incidence), the translational energies of scattered molecules tend to be slightly higher than $2 k_{B} T_{s}$, especially for large scattering angles $\theta_{f}$. This might be because the incident molecules with small grazing angles are more likely to be scattered back in the topmost layer before penetrating deep into the film and thereby to preserve their translational energies. The molecules scattered into large scattering angles, however, do not significantly contribute the accommodation coefficient of the overall energy transfer since the scattering flux for large angles is much less than that for small angles. For this reason, the accommodation coefficient remains high even for the large incident angles.

Table 1 summarizes the energy accommodation coefficients of He gas molecules on the quartz substrate and the

(a)

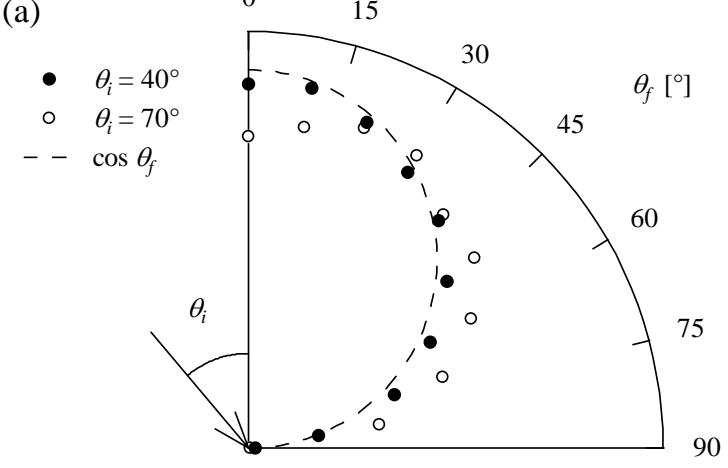

(b)

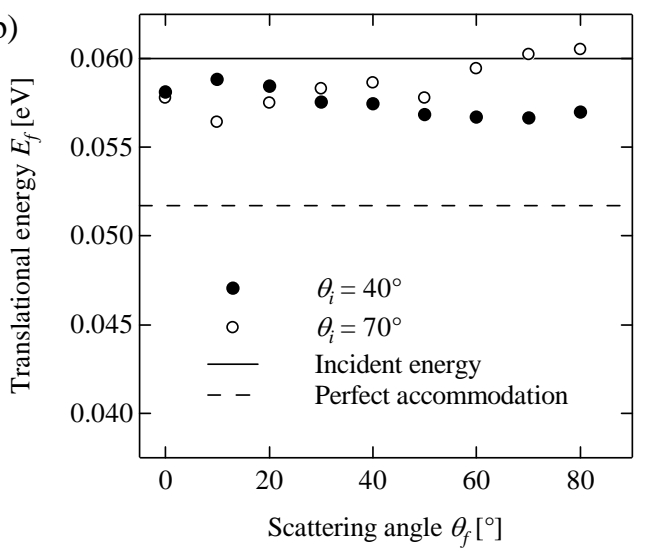

FIGURE 3. The scattering of He beam $\left(E_{i}=0.06 \mathrm{eV}\right)$ from the pre-growth quartz substrate with dispersed Co-Mo nanoparticles at room temperature. (a) The angular dependence of scattering intensities. (b) The mean translational energies of scattered molecules.

(a)

$$
\begin{aligned}
\text { - } & \theta_{i}=40^{\circ} \\
\square & \theta_{i}=60^{\circ} \\
\circ & \theta_{i}=70^{\circ} \\
-- & \cos \theta_{f}
\end{aligned}
$$

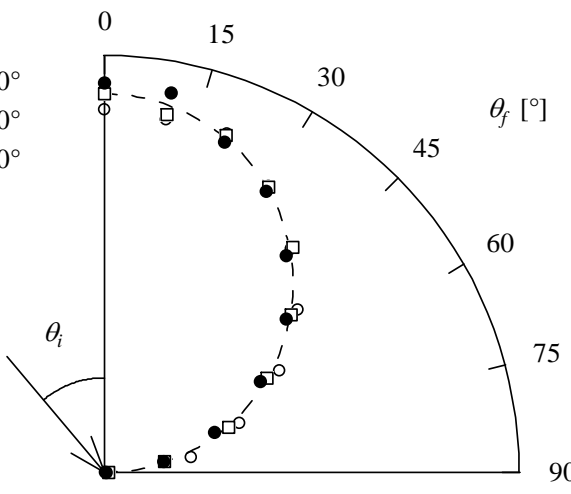

(b)

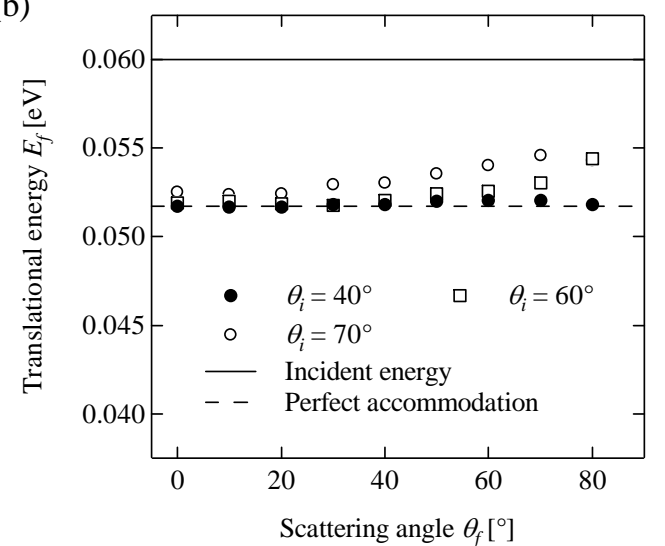

FIGURE 4. The scattering of He beam $\left(E_{i}=0.06 \mathrm{eV}\right)$ from the VA-SWNT film with a thickness of $4 \mu \mathrm{m}$ at room temperature. (a) The angular dependence of scattering intensities. (b) The mean translational energies of scattered molecules. 
VA-SWNT films deduced from our molecular beam experiments. The accommodation coefficients for other surfaces reported in literatures [8-10] are also included in this list. The relatively small accommodation coefficients except for the VA-SWNT films indicate that, in contrast to the heavier gas molecules, He molecules are hardly equilibrated with surface temperature through the scattering process even if the surface is covered with gas adsorbates or nanoparticles, which produce atomic-scale roughness significantly. With the Baule formula [16] in mind, we can partly attribute the low accommodation coefficients to the large mismatch between the mass of He molecules and the effective mass of the involved surface atoms. The surface modification with VA-SWNT films, however, increases remarkably the accommodation coefficients compared to those for bare surfaces. Although the accommodation coefficient slightly decreases for the thinner film, it would be still high enough for practical applications. The dependence of the accommodation coefficient on the film thickness implies that gas molecules can easily penetrate deep into the films because of their high porosity and suffer more than one collision with bundles of SWNTs.

The above discussion presents the effectiveness of the surface modification with VA-SWNT films for enhancing the energy transfer between gas molecules and surfaces at around room temperature. The question then arises as to whether this method is useful for higher temperature or not. Figure 5 shows the temperature dependence of the mean translational energy of scattered molecules to answer this question. As the surface temperature increases, the translational energy deviates far from that corresponding to the perfect accommodation. The less effective energy accommodation at elevated temperatures indicates that the average number of collisions which gas molecules undergo before leaving the film is not large enough to maintain the perfect accommodation even at high temperature. In order to elucidate the temperature dependence of the accommodation process, we estimated the potential energy well of He near SWNT bundles to be several hundredths of electronvolts by employing the potential model used for molecular dynamics simulations [5]. Since the potential well is of the same order as the thermal energy $\sim k_{B} T_{s}$ at room temperature, we would expect the significant decrease in the trapping probability of He molecules on SWNT bundles with increasing temperature and, as a consequence, the less effective energy exchange during every single collision.

TABLE 1. The energy accommodation coefficients $\alpha$ of He gas molecules on surfaces.

\begin{tabular}{lcl}
\hline Surface & Temperature & $\alpha$ \\
\hline Quartz substrate with Co-Mo catalyst nanoparticles & Room temperature $(\sim 300 \mathrm{~K})$ & 0.34 \\
VA-SWNT film on quartz substrate $(4 \mu \mathrm{m}$ in thickness) & Room temperature $(\sim 300 \mathrm{~K})$ & 0.97 \\
VA-SWNT film on quartz substrate $(0.1 \mu \mathrm{m}$ in thickness) & Room temperature $(\sim 300 \mathrm{~K})$ & 0.82 \\
Nickel (gas covered) [8] & $273 \mathrm{~K}$ & 0.360 \\
Nickel (gas covered) [9] & Room temperature & 0.385 \\
Tungsten (clean) [10] & $331 \mathrm{~K}$ & 0.0186 \\
Tungsten (gas covered) [9] & Room temperature & 0.470 \\
Platinum (gas covered) [8] & $273 \mathrm{~K}$ & 0.17 \\
Platinum (gas covered) [9] & Room temperature & 0.368 \\
\hline
\end{tabular}

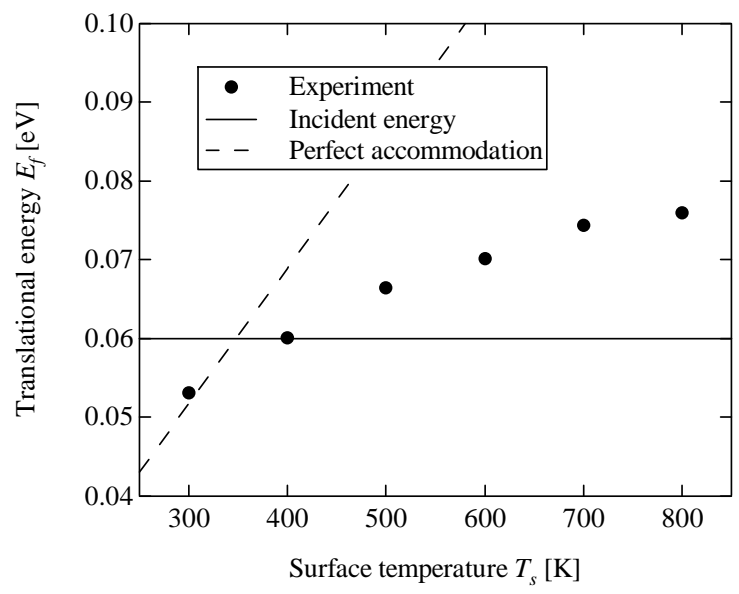

FIGURE 5. The mean translational energy of $\mathrm{He}\left(E_{i}=0.06 \mathrm{eV}, \theta_{i}=40^{\circ}\right)$ scattered from the VA-SWNT film with a thickness of $0.1 \mu \mathrm{m}$ as a function of the surface temperature. 


\section{CONCLUSION}

We have performed scattering experiments of He molecules on vertically aligned single-walled carbon nanotubes (VA-SWNTs) using the molecular beam technique. The accommodation coefficients for VA-SWNT films on quartz substrates at room temperature are remarkably high compared to those for bare surfaces, demonstrating the effectiveness of the surface modification technique with VA-SWNT films for enhancing the energy transfer between gas molecules and surfaces. The enhanced energy transfer suggests that gas molecules can easily penetrate deep into the films because of their high porosity and suffer multiple collisions with bundles of SWNTs. The less effective energy accommodation at elevated temperatures, however, implies that the average number of collisions which gas molecules undergo before leaving the films is not large enough to maintain the perfect accommodation even at high temperature.

\section{REFERENCES}

1. S. Iijima and T. Ichihashi, Nature 363, 603-605 (1993).

2. R. Saito et al., Physical Properties of Carbon Nanotubes, London: Imperial College Press, 1998.

3. Y. Murakami et al., Chem. Phys. Lett. 385, 298-303 (2004).

4. For example, H. Ulbricht et al., Carbon 44, 2931-2942 (2006).

5. K. Bolton and S. Gustavsson, Chem. Phys. 291, 161-170 (2003); K. Bolton and A. Rosén, Phys. Chem. Chem. Phys. 4, 44814488 (2002).

6. R. Xiang et al., J. Phys. Chem. C 112, 4892-4896 (2008).

7. G. Scoles ed., Atomic and Molecular Beam Methods Vol. 1, New York: Oxford University Press, 1988.

8. P. Rolf, Phys. Rev. 65, 185-189 (1944).

9. I. Amdur and L. A. Guildner, J. Am. Chem. Soc. 79, 311-315 (1957).

10. D. V. Roach and L. B. Thomas, "Determination of Thermal Accommodation Coefficients of Gases on Clean Surfaces at Temperatures Above $300 \mathrm{~K}$ by the Temperature Jump Method” in Fifth Symposium on Rarefied Gas Dynamics, edited by C. L. Brundin, New York: Academic Press, 1967, Vol. 1, pp. 163-172.

11. E. Einarsson et al., J. Phys. Chem. C 111, 17861-17864 (2007).

12. I. Kinefuchi et al., "Out-of-plane Scattering Distribution of Nitrogen Molecular Beam on Graphite (0001) Surface” in Rarefied Gas Dynamics: 24th International Symposium on Rarefied Gas Dynamics, edited by M. Capitelli, AIP Conference Proceedings 762, American Institute of Physics, Melville, NY, 2005, pp. 947-952.

13. Y. Murakami and S. Maruyama, Chem. Phys. Lett. 422, 575-580 (2006).

14. J. E. Hurst et al., Phys. Rev. Lett. 43, 1175-1177 (1979).

15. M. Hu et al., J. Catal. 225, 230-239 (2004).

16. J. Harris, "Mechanical Energy Transfer in Particle-Surface Collisions,” in Dynamics of Gas-Surface Interactions, edited by C. T. Rettner and M. N. R. Ashfold, Cambridge: Royal Society of Chemistry, 1991, pp. 1-46. 